\title{
OPTIMIZACIÓN DINÁMICA DE PLANTAS DE TRATAMIENTO DE AGUAS RESIDUALES USANDO CONTROL PREDICTIVO ECONÓMICO
}

\author{
Silvana Revollar Chávez \\ Dpto. de Informática y Automática, Universidad de Salamanca. España. srevolla@usal.es \\ Pastora Vega Cruz \\ Dpto. de Informática y Automática, Universidad de Salamanca. España. pvega@usal.es \\ Mario Francisco \\ Dpto. de Informática y Automática, Universidad de Salamanca. España.mfs@usal.es \\ Rosalba Lamanna \\ Dpto. de Procesos y Sistemas, Universidad Simón Bolívar. Venezuela. lamanna@usb.ve \\ Rosa García Vicente \\ Dpto. de Informática y Automática, Universidad de Salamanca. España. gvrosam@hotmail.com
}

\begin{abstract}
Resumen
En este trabajo, se propone la aplicación de una estrategia de control predictivo económico de una sola capa para realizar la optimización dinámica de estaciones de tratamiento de aguas residuales (EDAR). Se ha implementado un algoritmo de control predictivo económico no lineal (EMPC) con una función de coste que minimiza los costes de operación y la penalización de la desviación de la concentración de compuestos amoniacales en el efluente aprovechando la variabilidad de la carga del afluente. La operación se mantiene alrededor del punto de referencia que garantiza el rendimiento del sistema, debido a las restricciones consideradas sobre las variables controladas, pero estas se conducen a puntos de funcionamiento alternativos que optimizan la economía cuando las condiciones de proceso son favorables.
\end{abstract}

Palabras clave: Controlador predictivo económico, optimización dinámica, plantas de tratamiento de aguas.

\section{INTRODUCCIÓN}

El propósito de las instalaciones de tratamiento de aguas residuales urbanas es reducir la concentración de contaminantes del afluente a los niveles permitidos por la ley para ser descargadas en los distintos cuerpos de agua.

Las características del afluente son determinadas por los hábitos de consumo de agua de la población a lo largo de un día, las estaciones y cambios climáticos.
La eliminación biológica de nutrientes y materia orgánica se lleva a cabo en el proceso de lodos activados, que es el proceso más importarte de una planta típica de tratamiento de aguas residuales (EDAR). Los procesos biológicos involucrados en el tratamiento del agua se ven afectados por las variaciones en la actividad microbiana en invierno o verano y por las variaciones en la carga durante las estaciones lluviosas o secas. Sin embargo, la mayoría de las EDAR aplica estrategias conservadoras que regulan el tratamiento biológico más importante (procesos de fangos activados) alrededor del punto de funcionamiento nominal, aunque en los periodos de menor carga es posible alcanzar la calidad del efluente deseada en condiciones de operación que implican un menor consumo de energía [12].

Una solución atractiva para mejorar el funcionamiento de las EDAR es la implementación de estrategias de control que ajusten las condiciones operación de la planta a la carga actual del afluente considerando un periodo de supervisión adecuado para capturar estas variaciones y realizar los cambios necesarios en las consignas de las variables controladas [11], [12], [14].

En la mayoría de los procesos industriales se emplean arquitecturas de control jerárquico para la optimización sistemática del punto de operación. Estas estrategias generalmente se implementan considerando la política de operación en estado estacionario, realizando la optimización en tiempo real (RTO) de las consignas que se envían al nivel de control [10]. En el proceso de lodos activados de una EDAR, la frecuencia de los cambios del afluente dificulta el logro de condición de estado estacionario, entonces las plantas exhiben comportamientos 
dinámicos no estacionarios. Por lo tanto, para mejorar la operación de la planta y aprovechar las variaciones diarias del perfil del afluente para mejorar la economía, las estrategias de optimización en tiempo real deben adaptarse a las características particulares de las EDAR. La integración de la optimización económica del punto de operación en el algoritmo de un controlador predictivo (MPC) es una solución atractiva para realizar la optimización dinámica de la operación [4], [5].

En la literatura [10], [3], [14], [13] se encuentran aplicaciones exitosas de la optimización de consignas considerando la operación en estado estacionario y la operación dinámica. Sin embargo, el uso de estructuras compactas de una sola capa que actúen directamente sobre las variables manipuladas para lograr condiciones de operación óptimas es atractivo para evitar la complejidad de las arquitecturas jerárquicas.

En general, los EMPCs son una modificación del algoritmo de control predictivo (MPC) estándar que permite incluir objetivos económicos en la función de coste. Algunos autores [6], [9] hacen una distinción el EMPC puro, que utiliza una función de coste basada exclusivamente en el criterio económico y el controlador EMPC de seguimiento de referencia orientado a la economía (Hybrid-EMPC) que incluye índices de desempeño económico y de control en la función objetivo. Ambas técnicas son interesantes para realizar la optimización dinámica de la economía en los casos procesos que enfrentan cambios frecuentes en las entradas en donde la optimización de consignas tradicional basada en la suposición de operación en estado estacionario no es realista (Ellis et al., 2014)

En la literatura se encuentran varias aplicaciones de EMPCs, ya sea como optimizador de consignas en una estructura jerárquica o directamente en el nivel de control [1], [4], [7], [5]. Aplicaciones particulares de EMPC a las EDAR se encuentran en [11], [12], [14], [15].

Vega et al. [14] compara varias estructuras multicapa para optimizar la operación de la EDAR; dos de las estructuras propuestas incluyen un EMPC para la optimización dinámica de los puntos de referencia. Zeng and Liu [15] propuso una formulación particular del controlador predictivo económico implementando en una estructura de una sola capa, para mejorar la calidad de los efluentes y los costes de operación de la planta.

En este trabajo, se propone una estrategia de control predictivo económico de una sola capa para optimizar a operación del proceso de fangos activados afectando directamente las variables manipuladas. Es una alternativa prometedora a las estructuras jerárquicas, que evita el uso de diferentes modelos en las diferentes capas y proporciona una reacción más rápida a las perturbaciones.

\section{DESCRIPCIÓN DEL PROCESO}

Las estaciones depuradoras de aguas residuales (EDAR) comprenden diferentes etapas que constan de tratamientos biológicos, físicos y físico-químicos para la eliminación de contaminantes. El proceso de lodos activados es el tratamiento biológico más común utilizado en las EDARs municipales para logar la calidad requerida del efluente. La degradación de la materia orgánica y la eliminación de nutrientes se realiza mediante los procesos de nitrificación y desnitrificación. El crecimiento de microorganismos autótrofos en condiciones aeróbicas produce la transformación de amonio a nitratos (nitrificación). La reducción de nitratos a nitrógeno gaseoso $\left(\mathrm{N}_{2}\right)$ se produce en condiciones anóxicas debido a la actividad de microorganismos heterótrofos.

La plataforma de simulación "Benchmark Simulation Model (BSM1)" representa el proceso de lodos activados. Una descripción detallada del BSM1 incluyendo los procesos biológicos, perfiles de afluentes, parámetros cinéticos se presenta en [2]. El esquema del BSM1 consiste en dos reactores anóxicos y tres reactores aeróbicos. La plataforma BSM1 usa el modelo "activated sludge model Nol (ASM1)" para la descripción matemática del comportamiento de los biorreactores.

En este trabajo se utiliza un modelo reducido del BSM1 que contiene un reactor anóxico y otro aireado de volumen equivalente, como se muestra en la figura 1.

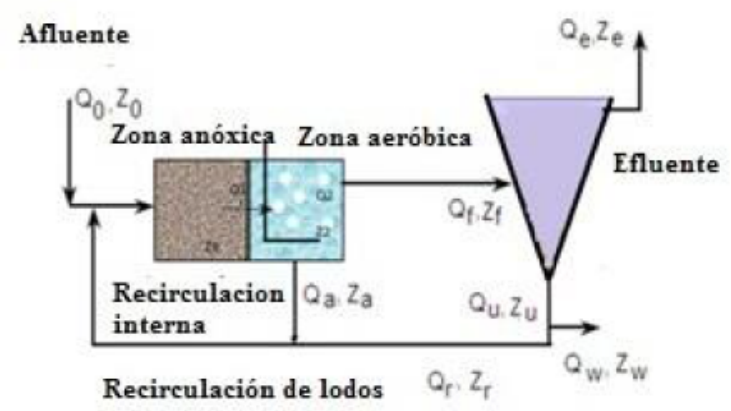

Figura 1: Representación esquemática del modelo BSM1 reducido.

Además, el modelo BSM1 se ha reducido para representar sólo los procesos biológicos más rápidos. Entonces los procesos lentos (la hidrólisis de las moléculas grandes) no se tienen en cuenta y la concentración de la biomasa autótrofa y heterótrofa se consideran constantes.

Los procesos biológicos considerados en el modelo reducido son el crecimiento de la biomasa heterótrofa en 
condiciones aeróbicas $\left(\rho_{1 i}\right)$, el crecimiento de la biomasa heterótrofa en condiciones anóxicas $\left(\rho_{2 i}\right)$ y el crecimiento de la biomasa autótrofa en condiciones aeróbicas $\left(\rho_{3 i}\right)$. En [2] se encuentra la representación matemática de los procesos biológicos, así como los valores típicos de los parámetros para una temperatura alrededor de $15^{\circ} \mathrm{C}$. Una lista de los parámetros del proceso y las variables consideradas en el modelo reducido usado aquí se muestran en la tabla 1 .

Tabla 1: Variables y parámetros del modelo reducido BSM1

\begin{tabular}{|c|c|c|}
\hline Descripción & Nombre & Valor \\
\hline \multicolumn{3}{|l|}{ Variables del proceso } \\
\hline Volumen del reactor anóxico $\left(\mathrm{m}^{3}\right)$ & $\overline{V_{1}}$ & 2000 \\
\hline Volumen del reactor aerobic $\left(\mathrm{m}^{3}\right)$ & $V_{2}$ & 3999 \\
\hline $\begin{array}{l}\text { Concentración de biomasa } \\
\text { heterótrofa activa }\left(\mathrm{gr} / \mathrm{m}^{3}\right)\end{array}$ & $X_{B . H}$ & 2500 \\
\hline $\begin{array}{l}\text { Concentración de biomasa } \\
\text { autótrofa activa }\left(\mathrm{gr} / \mathrm{m}^{3}\right)\end{array}$ & $X_{B, A}$ & 150 \\
\hline $\begin{array}{c}\text { Concentración de oxígeno } \\
\text { disuelto }\left(\mathrm{gr} / \mathrm{m}^{3}\right)\end{array}$ & So & $0.1-5$ \\
\hline $\begin{array}{c}\text { Concentración de nitratos } y \\
\text { nitritos }\left(\mathrm{gr} / \mathrm{m}^{3}\right)\end{array}$ & $S_{N O}$ & $0.1-5$ \\
\hline $\begin{array}{c}\text { Concentración } \mathrm{NH}_{4}^{+}+\mathrm{NH}_{3} \\
\left(\mathrm{gr} / \mathrm{m}^{3}\right)\end{array}$ & $S_{N H}$ & $0-14$ \\
\hline $\begin{array}{c}\text { Concentración de materia } \\
\text { orgánica }\left(\mathrm{gr} / \mathrm{m}^{3}\right)\end{array}$ & $S_{S}$ & $0-40$ \\
\hline Caudal afluente $\left(\mathrm{m}^{3} / \mathrm{h}\right)$ & $Q_{\text {in }}$ & $45-2500$ \\
\hline Caudal efluente $\left(\mathrm{m}^{3} / \mathrm{h}\right)$ & $Q_{e}$ & $45-2500$ \\
\hline $\begin{array}{c}\text { Concentración de material } \\
\text { orgánico en el afluente }\left(\mathrm{gr} / \mathrm{m}^{3}\right)\end{array}$ & $S_{S, \text { in }}$ & $45-140$ \\
\hline $\begin{array}{c}\text { Concentración de compuestos de } \\
\text { amonio en el afluente }\left(\mathrm{gr} / \mathrm{m}^{3}\right)\end{array}$ & $S_{N H, i n}$ & $8-55$ \\
\hline $\begin{array}{c}\text { Caudal de recirculación } \\
\text { interna }\left(\mathrm{m}^{3} / \mathrm{h}\right)\end{array}$ & $Q_{a}$ & $180-200$ \\
\hline $\begin{array}{c}\text { Coeficiente de transferencia de } \\
\text { oxigeno }(1 / \mathrm{h})\end{array}$ & $K L a$ & $0-10$ \\
\hline \multicolumn{3}{|c|}{ Parámetros del proceso biológico } \\
\hline $\begin{array}{c}\text { Concentración de saturación de } \\
\text { oxígeno }\left(\mathrm{gr} / \mathrm{m}^{3}\right)\end{array}$ & So,sat & 8 \\
\hline $\begin{array}{l}\text { Tasa maxima de crecimiento } \\
\text { heterótrofo especifico }(1 / d)\end{array}$ & $\mu_{H}$ & 4 \\
\hline $\begin{array}{c}\text { Coeficiente medio de saturación } \\
\text { para heterótrofos }\left(\mathrm{gr} / \mathrm{m}^{3}\right)\end{array}$ & Ks & 10 \\
\hline $\begin{array}{l}\text { Coeficiente de saturación de } \\
\text { oxigeno para heterótrofos }\left(\mathrm{gr} / \mathrm{m}^{3}\right)\end{array}$ & $K_{O, H}$ & 0.2 \\
\hline $\begin{array}{c}\text { Coeficiente de saturación de } \\
\text { amonios para heterótrofos } \\
\left(\mathrm{gr} / \mathrm{m}^{3}\right)\end{array}$ & $K_{N H}$ & 1 \\
\hline $\begin{array}{l}\text { Coeficiente de saturación de } \\
\text { oxígeno para autótrofos }\left(\mathrm{gr} / \mathrm{m}^{3}\right)\end{array}$ & $K_{O, A}$ & 0.4 \\
\hline Rendimiento heterotrófico & $Y_{H}$ & 0.67 \\
\hline Rendimiento autotrófico & $Y_{A}$ & 0.24 \\
\hline $\begin{array}{l}\text { Fracción de nitrógeno en la } \\
\text { biomasa }\end{array}$ & $i_{X B}$ & 0.08 \\
\hline
\end{tabular}

\subsection{MODELO}

A continuación, se presentan las ecuaciones del modelo matemático. Las variables del afluente consideradas en el modelo reducido son: caudal del afluente $\left(Q_{i n}\right)$, concentración de materia orgánica $\left(S_{S, i n}\right)$ y concentración de amonios $\left(S_{N H, i n}\right)$. Los estados asociados a los procesos biológicos más rápidos son: la concentración de los compuestos $\mathrm{NH}_{4}{ }^{+}+\mathrm{NH}_{3}\left(\mathrm{~S}_{\mathrm{NHi}}\right)$, la concentración de nitratos $\left(\mathrm{S}_{\mathrm{NO} i}\right)$, la concentración de la materia orgánica $\left(S_{S i}\right)$ y la concentración de oxígeno $(\mathrm{SO})$. El índice es usado para distinguir las unidades, siendo el reactor 1 la zona anóxica $(i=1)$ y el reactor 2 la zona aeróbica $(i=2)$. Las entradas son el coeficiente de transferencia de oxígeno $(K L a)$ ) y el caudal de recirculación interna $(Q a)$.

Procesos biológicos:

$$
\begin{aligned}
& \rho_{1 i}=\mu_{H} \cdot\left(\frac{S_{S i}}{K_{s}+S_{S i}}\right) \cdot\left(\frac{S_{O i}}{K_{O, H}+S_{O i}}\right) X_{B, H} \\
& \rho_{2 i}=\mu_{H} \cdot\left(\frac{S_{S i}}{K_{s}+S_{S i}}\right) \cdot\left(\frac{K_{O, H}}{K_{O, H}+S_{O i}}\right)\left(\frac{S_{N O i}}{K_{N O}+S_{N O i}}\right) n_{g} X_{B, H} \\
& \rho_{3 i}=\mu_{A} \cdot\left(\frac{S_{N H i}}{K_{N H}+S_{N H i}}\right) \cdot\left(\frac{S_{O i}}{K_{O, A}+S_{O i}}\right) X_{B, A}
\end{aligned}
$$

Ecuaciones diferenciales obtenidas de los balances de masas:

$$
\begin{aligned}
& \frac{d S_{N H 1}}{d t}=\frac{1}{V_{1}}\left[Q_{i n} \cdot S_{N H m}+Q_{a} S_{N H 2}-\left(Q_{i n}+Q_{a}\right) \cdot S_{N H 1}\right] \\
& -i_{X b} \cdot \rho_{11}-i_{X b} \cdot \rho_{21}-\left(i_{X b}+\frac{1}{Y_{A}}\right) \cdot \rho_{31} \\
& \frac{d S_{N O 1}}{d t}=\frac{1}{V_{1}}\left[Q_{a} S_{N O 2}-\left(Q_{i n}+Q_{a}\right) S_{N O 1}\right]
\end{aligned}
$$

$-\frac{1-Y_{H}}{2.86 Y_{H}} \rho_{21}+\frac{1}{Y_{A}} \cdot \rho_{31}$

$\frac{d S_{S 1}}{d t}=\frac{1}{V_{1}}\left[Q_{i n} \cdot S_{S i n}+Q_{a} S_{S 2}-\left(Q_{i n}+Q_{a}\right) S_{S 1}\right]$

$-\frac{1}{Y_{H}} \rho_{11}-\frac{1}{Y_{H}} \rho_{21}$

$\frac{d S_{O 1}}{d t}=\frac{1}{V_{1}}\left[Q_{a} \cdot S_{O 2}-\left(Q_{0}+Q_{a}\right) S_{O 1}\right]$

$-\left[\frac{1-Y_{H}}{Y_{H}} \rho_{11}+\left(\frac{4.57}{Y_{A}}+1\right) \rho_{31}\right]$

$\frac{d S_{N H 2}}{d t}=\frac{1}{V_{2}}\left[\left(Q_{i n}+Q_{a}\right) S_{N H 1}-\left(Q_{i n}+Q_{a}\right) S_{N H 2}\right]$

$-i_{x b} \cdot \rho_{12}-\left(i x_{b}+\frac{1}{Y_{A}}\right) \cdot \rho_{32}$

$\frac{d S_{\mathrm{NO} 2}}{d t}=\frac{1}{V_{2}}\left[\left(Q_{i n}+Q_{a}\right) S_{\mathrm{NO} 1}-\left(Q_{i n}+Q_{a}\right) S_{\mathrm{NO} 2}\right]$

$-\frac{1-Y_{H}}{2.86 Y_{H}} \rho_{22}+\frac{1}{Y_{A}} \cdot \rho_{32}$

$\frac{d S_{S 2}}{d t}=\frac{1}{V_{2}}\left[\left(Q_{i n}+Q_{a}\right) S_{S 1}-\left(Q_{i n}+Q_{a}\right) S_{S 2}\right]$

$-\frac{1}{Y_{H}} \rho_{12}-\frac{1}{Y_{H}} \rho_{22}$ 


$$
\begin{aligned}
& \frac{d S_{O 2}}{d t}=\frac{1}{V_{2}}\left[\left(Q_{i n}+Q_{a}\right) \cdot S_{O 1}-\left(Q_{i n}+Q_{a}\right) \cdot S_{O 2}\right] \\
& -\left[\frac{1-Y_{H}}{Y_{H}} \rho_{12}+\frac{4.57-Y_{A}}{Y_{A}} \rho_{32}\right]+K \operatorname{KL}\left(S_{O, \text { sat }}-S_{O 2}\right)
\end{aligned}
$$

\subsection{CARACTERÍSTICAS DEL AFLUENTE}

La plataforma del BSM1 proporciona datos del afluente para diferentes climas que representan los picos y valles de la carga a lo largo del día, y eventos meteorológicos como lluvia y tormentas [2]. Para evaluar la estrategia de control propuesta se ha diseñado un perfil particular del afluente para una ventana de operación de ocho días usando el perfil de tiempo seco del BSM1, un fin de semana con actividad humana reducida y un evento lluvioso (figura 2). En un día típico del perfil (día 2 o el día 6) se observa un periodo de menor carga (en la noche), un pico en la mitad de la mañana y un periodo de carga media (por la tarde). El evento de lluvia entre los días 3 y 5 , produce un gran incremento en el caudal del afluente $\left(Q_{i n}\right)$, y disminuye la concentración de materia orgánica $\left(S_{S, \text { in }}\right)$ y de amonios $\left(S_{N H, i n}\right)$. Este perfil de afluente combina perturbaciones medias y rápidas en un tiempo comprendido entre horas y días.

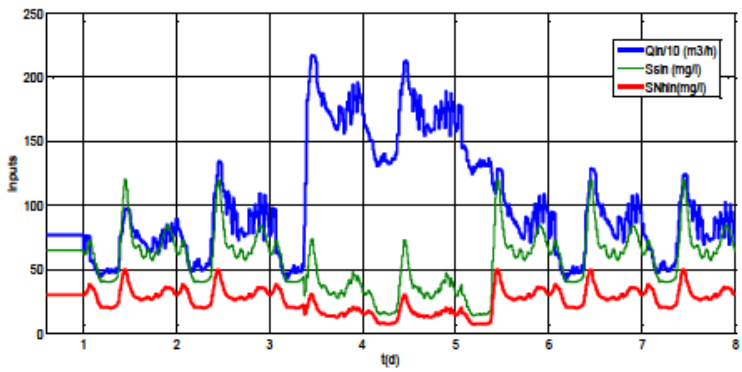

Figura 2: Perfil del afluente durante 8 días de operación.

\section{PROBLEMA DE CONTROL}

La formulación del problema de control depende de la influencia de las variables de control del proceso de lodos activados sobre el rendimiento general de la EDAR y sobre la calidad del efluente. La calidad del efluente está condicionada por los límites impuestos por la regulación medioambiental sobre la concentración de materia orgánica y nutrientes de las descargas de la EDAR. Los límites considerados para la calidad del efluente para el modelo BSM1 se muestran en la tabla 2.

Tabla 2: Límites de calidad del efluente.

\begin{tabular}{cc}
\hline Variable & Límite \\
\hline Nitrógeno total $(\mathrm{Nt})$ & $<18 \mathrm{grN} / \mathrm{m}^{3}$ \\
Demanda Química de Oxigeno & $<100 \mathrm{grCOD} / \mathrm{m}^{3}$ \\
(COD,e) & $<4 \mathrm{grN} / \mathrm{m}^{3}$ \\
Concentración de amonios $\left(S_{N H, e}\right)$ & $<10 \mathrm{grN} / \mathrm{m}^{3}$ \\
Concentración de nitratos $\left(S_{\mathrm{NO}, e}\right)$ & \\
\hline
\end{tabular}

En los procesos de lodos activados una variable clave es la concentración de oxígeno disuelto en la zona aeróbica $(\mathrm{SO} 2)$ ya que los microorganismos requieren suficiente oxígeno para eliminar los nutrientes y la materia orgánica a través del proceso de nitrificación. En la zona anóxica, la concentración de nitratos $\left(S_{\mathrm{NOl}}\right)$ tiene una influencia significativa en el proceso de nitrificación. Los detalles sobre el proceso de nitrificación y desnitrificación se pueden encontrar en [8].

En la plataforma BSM1 y en varias aplicaciones [14], [15] la estrategia de control se centra directa o indirectamente en la regulación de la concentración de oxígeno disuelto en la zona aireada $(\mathrm{SO} 2)$ y la regulación de la concentración de nitratos en la zona anóxica $\left(\mathrm{S}_{\mathrm{NOI}}\right)$ usando el coeficiente de transferencia de oxígeno $(K L a)$ y el caudal de recirculación interna ( $Q a)$ como variables manipuladas. El BSM1 aplica un esquema de control basado en dos lazos que usa controladores típicos PI (Proporcional Integral) [2].

Al definir el problema de optimización y control, se debe tomar en cuenta que las violaciones de los límites mostrados en la tabla 2 sobre la concentración de los contaminantes en el efluente tienen un impacto en la economía debido a las multas impuestas por los vertidos fuera de especificación.

En la operación convencional del proceso de fangos activados, la regulación de la concentración de oxígeno disuelto $\left(\mathrm{SO}_{2}\right)$ y nitratos $\left(\mathrm{S}_{\mathrm{NO} I}\right)$ a los valores de referencia requiere de una cantidad significativa de energía de aireación y de bombeo. Este tipo de operación no toma en cuenta que, en los periodos de menor carga, la disminución en los niveles de contaminantes facilita el tratamiento y las normas de calidad del efluente se logran incluso con valores de oxígeno disuelto $\left(\mathrm{SO}_{2}\right)$ por debajo de la referencia típica de $2 \mathrm{mg} / \mathrm{l}$. Por lo tanto, en estas condiciones, conducir esta variable al punto de referencia puede resultar en un consumo innecesario de energía. Por otro lado, durante los periodos de mayor carga es necesario aumentar la actividad biológica para reducir la cantidad excesiva de contaminantes, entonces, el logro del punto de referencia para $\mathrm{SO}_{2}$ y $S_{\mathrm{NO} 1}$ es decisivo para cumplir con las especificaciones de calidad del efluente o minimizar las violaciones de los límites impuestos para evitar sanciones económicas.

En este trabajo se considera una estrategia de control multivariable, donde el valor de referencia de la concentración de nitratos $\mathrm{S}_{\mathrm{NO} 1 \_\mathrm{SP}}=1.675 \mathrm{~g} / \mathrm{m}^{3}$ y la concentración de oxígeno disuelto $\mathrm{S}_{\mathrm{O} 2} \mathrm{SP}=2.384 \mathrm{~g} / \mathrm{m}^{3}$ han sido obtenidos a partir de la optimización de estos valores en estado estacionario para un periodo de operación de dos semanas. Las variables controladas $\left(\mathrm{SO}_{2}\right.$ y $\left.S_{\mathrm{NO} I}\right)$ se regulan utilizando los valores de variables manipuladas $(K L a)$ y $\left(Q_{a}\right)$ obtenidas usando una ley de control que considera simultáneamente el logro del punto de referencia y la minimización de los costes energéticos. Estos son objetivos conflictivos, pero, la flexibilidad del proceso es explotada para mejorar la economía en lugar de cumplir el logro del punto de referencia en las situaciones donde es posible. Los límites físicos son considerados para calcular las variables manipuladas, así como las variaciones 
admisibles de las variables controladas para asegurar condiciones seguras para el proceso y operación aceptable.

\section{1 ÍNDICES DE RENDIMIENTO}

En la representación reducida de ASP considerada en este trabajo, el coste de operación asociado a consumo de energía incluye sólo la energía de la aireación y la energía de bombeo, el cual se calcula utilizando los índices de costes propuestos en la plataforma BSM1 [2]. En el caso estudiado, la energía de bombeo (PE) representa el uso de energía para el bombeo del flujo de recirculación interna $(Q a)$ :

$$
P E=\frac{1}{T} \int_{t_{0}}^{t f}\left(0.004 \cdot Q_{a}(t)\right) d t[k W h / d]
$$

El coeficiente de transferencia de oxígeno $K L a$ es un parámetro el cual tiene en cuenta factores como el tipo de difusor en los reactores, el tamaño de la burbuja y la profundidad de inmersión. La energía de aireación (AE) es calculada a partir del $K L a$ según la siguiente ecuación:

$$
A E=\frac{S_{O, s a t}}{T \cdot 1.8 \cdot 1000} \int_{t_{0}}^{t f} V_{2} \cdot K L a(t) d t[k W h / d]
$$

$\mathrm{El}$ coste total (OCI) incluye la energía de bombeo (PE) y la energía de aireación (AE):

$$
O C I=\left(\begin{array}{l}
w_{1} \cdot \frac{1}{T} \int_{t_{0}}^{t}\left(0.004 \cdot Q_{a}(t)\right) d t \\
+w_{2} \cdot \frac{S_{0, \text { sat }}}{T \cdot 1.8 \cdot 1000} \int_{t_{0}}^{\delta} V_{2} \cdot K L a(t) d t
\end{array}\right)[E U R / d]
$$

$w_{1}$ y $w_{2}$ son factores de costes, se eligen $w_{1}=w_{2}=1$ EUR/kWh

El rendimiento de la planta se mide respecto a la calidad del efluente, y la calidad del efluente estimada considera sólo la concentación de compuestos de $\mathrm{NH}_{4}^{+}+\mathrm{NH}_{3}$ en el efluente $\left(S_{N H, e}\right)$. Esto supone que $S_{N H, e}$ es idéntico a $S_{N H 2}$ en la zona aeróbica. Los niveles deseados sobre el COD mostrados en la tabla 2 se satisfacen fácilmente, el nitrógeno total $\mathrm{Nt}$ incluye los compuestos de $\mathrm{NH}_{4}^{+}+$ $\mathrm{NH}_{3}$ y estos compuestos son más perjudiciales que los nitratos en el efluente $\left(S_{\mathrm{NO}, e}\right)$.

Se desea una concentración de compuestos de $\mathrm{NH}_{4}{ }^{+}+$ $\mathrm{NH}_{3}$ en el efluente $\left(S_{\mathrm{NH}, e}\right)$ por debajo de $4 \mathrm{mg} / 1$ sobre los valores medios diarios de $S_{N H, e}$ :

$$
S_{N H a v}=\frac{\int_{t_{0}}^{t_{0}+24 h} S_{N H, e}(t) \cdot Q_{e}(t) \cdot d t}{\int_{t_{0}}^{t_{0}+24 h} Q_{e}(t) \cdot d t}
$$

Un índice de desempeño que mide la multa económica por las violaciones de $S_{N H, e}$ es:

$$
\text { Cost_NH } H_{e f f}=\left\{\begin{array}{c}
0 \text { for } S_{N H, e}<4 \\
\frac{8.2}{1000} \int_{t_{0}}^{\pi}\left(S_{N H, e}(t)-4\right) \cdot Q_{e}(t) \cdot d t[E U R / d] \\
\text { for } S_{N H, e} \geq 4
\end{array}\right.
$$

El índice Cost_NHeff es una medida de penalización económica para las descargas fuera de especificación acumuladas en el periodo de operación. Cost_NHeff como una medida del desempeño del proceso (costes por violar la calidad del efluente) comparable con el índice del desempeño económico OCI, utilizado en este documento con fines comparativos. El índice se calcula considerando los valores instantáneos de $S_{N H, e}$, pero el efluente se considera fuera de especificación y se aplican multas, sólo cuando la concentración media diaria de $S_{N H, e}$ infringe los límites impuestos $S_{N H a v}$ $\geq 4 \mathrm{mg} / 1$

\section{FORMULACIÓN DEL PROBLEMA DE CONTROL CON EMPC}

La formulación general de los controladores de seguimiento orientados económicos se presenta en [5], [12]. La formulación particular para la planta de tratamiento de aguas utilizando el modelo seleccionado del proceso de lodos activados y los índices de desempeño que se acaban de describir se muestran a continuación.

Vector de estado:

$$
\mathbf{x}(t)=\left[\begin{array}{llllllll}
S_{N H 1} & S_{N H 2} & S_{N O 1} & S_{N O 2} & S_{S 1} & S_{S 2} & S_{O 1} & S_{O 2}
\end{array}\right]
$$

Variables de salida (variables controladas):

$$
\mathbf{y}(t)=\left[\begin{array}{ll}
S_{\mathrm{NO} 1} & S_{\mathrm{O} 2}
\end{array}\right]
$$

Perturbaciones de las medidas:

$$
\mathbf{v}(t)=\left[\begin{array}{lll}
Q_{i n} & S_{S, i n} & S_{N H, i n}
\end{array}\right]
$$

Vector de entradas manipulables:

$$
\mathbf{u}(t)=\left[\begin{array}{ll}
Q_{a} & K L a
\end{array}\right]
$$

- Modelo de predicción: el modelo de proceso nolineal descrito por las ecuaciones (1)-(11) es usado como predicción del modelo de forma $\dot{\hat{\mathbf{x}}}(t)=f(\hat{\mathbf{x}}(t), \mathbf{u}(t), \mathbf{v}(t))$ como se describe en [12]

\subsection{FUNCIÓN DE COSTE}

En el problema de control predictivo basado en modelos estándar, el desempeño se mide como la desviación de las variables controladas respecto a los puntos de referencia a lo largo del horizonte de predicción (coste de etapa) y al final del horizonte de predicción (penalización terminal), junto con los movimientos necesarios de las variables manipuladas. En esta aplicación, el índice estándar de rendimiento MPC (ec. 17) se expresa en términos del vector de variables de salida $\mathbf{y}(t)=\left[\begin{array}{ll}S_{\mathrm{NO}} & S_{\mathrm{O} 2}\end{array}\right]$ el cual se selecciona como los 
estados controlados desde el vector de estado $\mathbf{x}(t)$ y los puntos de referencia $\mathbf{y}_{\mathbf{S P}}=\left[\begin{array}{ll}S_{N O 1 \_S P} & S_{O_{2} \_S P}\end{array}\right]$. El otro término en $J_{N M P C}$ es una medida de los movimientos de las entradas manipuladas $\mathbf{u}(t)=\left[\begin{array}{ll}Q_{a} & K L a\end{array}\right]$ respecto al paso anterior $S_{\text {NOI_SP }}=1.67 \mathrm{mg} / 1$ y $S_{O}{ }_{-} S P=2.38 \mathrm{mg} / 1$.

$$
\begin{aligned}
& J_{N M P C}(\hat{y}(t), u(t))=\left|\hat{y}(t)-y_{S P}\right|_{W y}^{2} \\
& +\left|\mathbf{u}(t)-\mathbf{u}\left(\tau_{k-1}\right)\right|_{W u}^{2}+\left|\hat{y}(N)-y_{S P}\right|_{W \mathbf{n}}^{2}
\end{aligned}
$$

$\mathrm{Y}$ Wy es la matriz de peso Wx para los correspondientes estados controlados. Los puntos de referencia son valores óptimos computados para el comportamiento del proceso deseado en un tiempo de operación comprendido entre semanas y meses: $\mathrm{S}_{\mathrm{NO} 1 \_} \mathrm{SP}=1.67 \mathrm{mg} / 1$ y $\mathrm{So}_{2} \mathrm{SP}=2.38 \mathrm{mg} / 1$.

En la formulación del problema de control predictivo económico (EMPC), las variables manipuladas óptimas se obtienen considerando el rendimiento económico $\left(J_{E C O}\right)$. En este caso, se mide con el índice total de costes (OCI) descrito por la (ec.14), que incluye la energía de bombeo y de aireación y Cost_NHeff.

$$
J_{E C O}(\hat{y}(t), u(t))=\left(\begin{array}{l}
w_{1} \cdot \frac{1}{T} \int_{t_{0}}^{\forall}\left(0.004 \cdot Q_{a}(t)\right) d t \\
+w_{2} \cdot \frac{S_{\text {OSat }}}{T \cdot 1.8 \cdot 1000} \int_{t_{0}}^{\nabla} V_{2} \cdot K L a(t) d t
\end{array}\right)[E U R / d]
$$

donde $w_{1}=w_{2}=1 E U R / \mathrm{kWh}$

La importancia de cada objetivo en el híbrido coste de función viene dada por el peso $w_{E}$ and $w_{C}$.

-Restricciones: se imponen las siguientes restricciones sobre las entradas KLa y $Q a$ y las variables controladas $\mathrm{S}_{\mathrm{NO} 1}$ y $\mathrm{SO}$.

$$
\begin{gathered}
180 \leq Q_{a}(t) \leq 3850\left[\mathrm{~m}^{3} / \mathrm{h}\right] \forall \mathrm{t} \in\left[0, \tau_{N}\right) \\
1.5 \leq K l a(t) \leq 10[1 / \mathrm{h}] \forall \mathrm{t} \in\left[0, \tau_{N}\right) \\
0.1 \leq S_{N O 1}(t) \leq 5\left[\mathrm{~g} / \mathrm{m}^{3}\right] \forall \mathrm{t} \in\left[0, \tau_{N}\right) \\
0.5 \leq S_{O 2}(t) \leq 5\left[\mathrm{~g} / \mathrm{m}^{3}\right] \forall \mathrm{t} \in\left[0, \tau_{N}\right)
\end{gathered}
$$

En algunos casos, bajo fuertes perturbaciones, puede ser imposible para la planta satisfacer todas las restricciones, entonces, las ecuaciones (19-22) se formulan como limitaciones suaves.

\section{RESULTADOS}

El comportamiento de la planta con el EMPC y la estrategia por defecto del BSM1 (regulación de $\mathrm{So}_{2}$ y
$S_{\mathrm{NO} I}$ a los valores de referencia) se simuló a lo largo de 8 días de operación considerando el perfil de perturbaciones de la figura 2. Para evaluar el desempeño del EMPC se consideraron valores extremos de los pesos de la función de coste descrita por la ec. 18. Los resultados obtenidos se presentan en las figuras 3 a 7 .

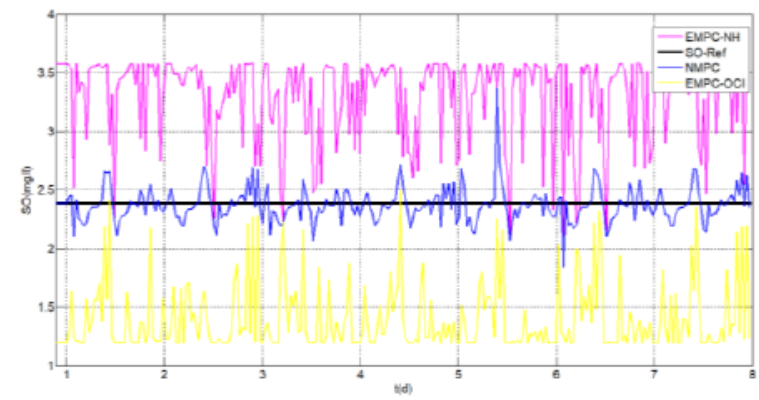

Figura 3: Comparación de la respuesta de $\mathrm{SO} 2$ con el EMPC propuesto considerando únicamente el coste económico (EMPC-OCI) y considerando únicamente Cost $N H_{\text {eff }}$ (EMPC-NH).

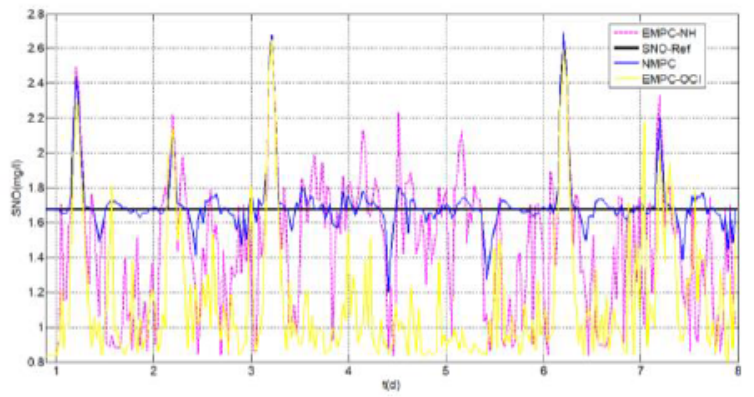

Figura 4: Comparación de la respuesta de $\mathrm{SNO} 1$ con el EMPC propuesto considerando únicamente el coste económico (EMPC-OCI) y considerando únicamente Cost_NH $H_{\text {eff }}(\mathrm{EMPC}-\mathrm{NH})$.

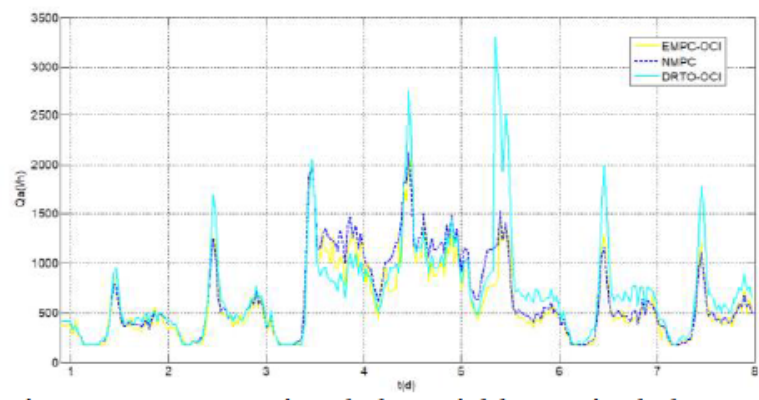

Figura 5: Comparación de la variable manipulada Qa con el EMPC propuesto considerando únicamente el coste económico (EMPC-OCI) y considerando únicamente Cost_NH eff $(\mathrm{EMPC}-\mathrm{NH})$.

En las figuras 3 y 4 se puede observar como el EMPC produce valores de respuesta que tienen a los valores mínimos de las variables controladas cuando se considera únicamente la optimización de objetivos económicos (EMPC-OCI). Estos valores corresponden al mínimo uso posible de energía de aireación asociado con la variable manipulada KLa y energía de bombeo asociado a Qa (figura 5 y 6). En cambio, cuando se 
optimiza únicamente la penalización económica debido a desviaciones de la concentración de amonio respecto al máximo valor permitido (EMPC-NH), se alcanzan los valores máximos permitidos de las variables controladas So2 y $S_{\mathrm{NO}}$. En ambos casos, la formulación del EMPC toma en cuenta las restricciones sobre las variables controladas que aseguran la calidad del efluente deseada.

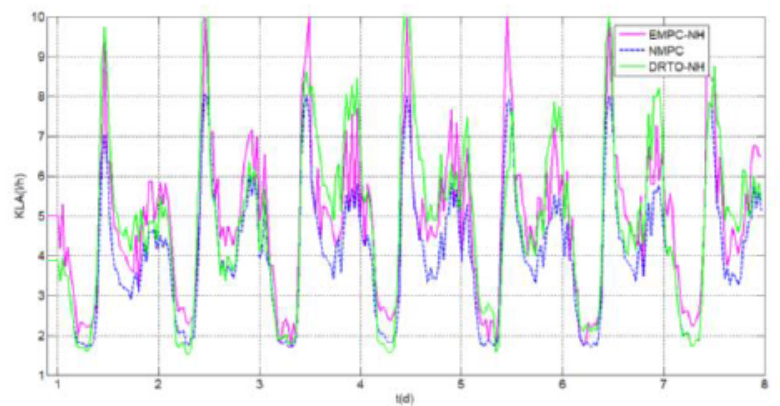

Figura 6: Comparación de la variable manipulada

$\mathrm{KLa}$ con el EMPC propuesto considerando

únicamente el coste económico (EMPC-OCI) y considerando únicamente Cost $N H_{\text {eff }}(\mathrm{EMPC}-\mathrm{NH})$.

\section{CONCLUSIONES}

La aplicación de la estrategia de control predictivo económico no lineal (EMPC) ha sido efectiva para optimizar dinámicamente la operación de la planta de tratamiento de aguas. Al comparar esta estrategia de control considerando, por una parte la minimización del coste económico, y por otra la minimización de la desviación de la concentración de compuestos amoniacales en el efluente, la operación se mantiene dentro de las condiciones que garantizan el rendimiento del sistema pero las variables controladas se conducen a puntos de funcionamiento alternativos que optimizan estos objetivos aprovechando la variabilidad de la carga del afluente. En ambos casos, la formulación del EMPC toma en cuenta las restricciones sobre las variables controladas que aseguran la calidad del efluente deseada.

\section{Agradecimientos}

Los autores agradecen el apoyo del Gobierno Español a través del Proyecto MINECO/FEDER DPI2015-67341-C2-1 y a los doctores Dr Ulf Jeppsson, Dr Christian Rosen y colaboradores por la plataforma BSM1.

\section{English summary}
DYNAMIC OPTIMIZATION OF THE OPERATION OF WASTEWATER TREATMENT PLANTS USING

\section{ECONOMIC MODEL PREDICTIVE CONTROL}

\begin{abstract}
In this paper, a single-layer economic model predictive control $(E M P C)$ strategy is presented. The objective of the EMPC is to perform the dynamic optimization of the operation of a wastewater treatment plant (WWTP). A non-linear EMPC algorithm has been implemented. The controller cost function represents the operating costs and the violations of maximum admitted levels of ammonium concentration in the effluent. The proposed strategy takes advantage of the variability of the influent load to find alternative operating points that minimize the cost function. Constraints are imposed on critical variables to ensure the conditions that guarantee the appropriate process performance.
\end{abstract}

Keywords: Economic model predictive controller, dynamic optimization, wastewater treatment plants.

\section{Referencias}

[1] Adetola, V.; Guay, M. (2010). Integration of real-time optimization and model predictive control. Journal of Process Control, 20, 125133.

[2] Alex, J.; Benedetti, L.; Copp, J.; Gernaey, K.; Jeppsson, U.; Nopens, I.; Pons, M.; Rieger, L.; Rosen, C.; Steyer, J.; Vanrolleghem, P. y Winkler, S. Benchmark Simulation Model no. 1 (BSM1). (2008). IWA Taskgroup on benchmarking of control strategies for WWTPs. Dpt. of Industrial Electrical Engineering and Automation, Lund University. Cod.: LUTEDXTEIE 7229. 1-62.

[3] Brdys, M.A., Grochowski, M., Gminski, T., Konarczak, K., Drewa, M. (2008) Hierarchical predictive control of integrated wastewater treatment systems, Control Eng. Pract. 16 (6), 751-767.

[4] Ellis, M. and Christofides, P. (2014) Integrating dynamic economic optimization and model predictive control for optimal operation of nonlinear process systems. Control Engineering Practice, 22: 242-251.

[5] Ellis, M.; Duranda, H.; Christofides, P. (2014) A tutorial review of economic model predictive control methods. Journal of Process Control. 24, 1156-1178

[6] Idris, E. A. N.; Engell, S. (2012) Economicsbased NMPC strategies for the operation and 
control of a continuous catalytic distillation process. J. Process Control. 22, 1832-1843.

[7] Limon, D.; Pereira, M.; Muñoz de la Peña, D.; Alamo, T.; Grosso, J.M. (2014) Single-layer economic model predictive control for periodic operation. Journal of Process Control, 24 (8), 1207-1224

[8] Makinia, J. (2010) Mathematical modeling and computer simulation of activated sludge systems. IWA publishing. London.

[9] Ochoa, S. G. Wozny, G. and Repke,J.U. (2010) Plantwide Optimizing Control of a continuous bioethanol production process. Journal of process Control. 20 (9), 983-998.

[10] Piotrowski R., M.A. Brdys, K. Konarczak, K. Duzinkiewicz and W. Chotkowski. (2008) Hierarchical dissolved oxygen control for activated sludge processes. Control Engineering Practice. 16 (1), 114-131.

[11] Revollar, S.; Vega, P.; Vilanova, R.; M. Francisco; I. Santín. (2016) Optimization of Economic and Environmental Objectives in a Non Linear Model Predictive Control Applied to a Wastewater Treatment Plant. 20th ICSTCC Rumania, octubre, 2016

[12] Revollar, S.; Vega, P.; Vilanova, R.; Francisco, M. (2017). Optimal Control of Wastewater Treatment Plants Using Economic-Oriented Model Predictive Dynamic Strategies". Applied Sciences 7(8):813. DOI: 10.3390/app7080813

[13] Santín, I.;. Pedret, C.; Vilanova. R. (2015b) Applying variable dissolved oxygen set point in a two level hierarchical control structure to a wastewater treatment process. Journal of Process Control. 28, 40-55

[14] Vega, P.; Revollar, S.; Francisco, M.; Martin, J. M. (2014) Integration of set point optimization techniques into nonlinear MPC for improving the operation of WWTPs. Comput. Chem. Eng. $68,78-95$.

[15] Zeng J.; Liu, J. (2015) Economic model predictive control of wastewater treatment processes. Industrial \& Engineering Chemistry Research, 54, ${ }^{\circ} 5710-5721$.

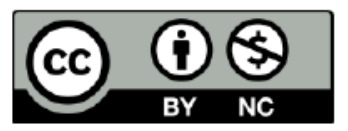

(c) 2018 by the authors. Submitted for possible open access publication under the terms and conditions of the Creative Commons Attribution CC-BY-NC 3.0 license (https://creativecommons.org/licenses/by-nc/3.0). 